\title{
Aplicación de un modelo para la implementación de logística inversa en la etapa productiva
}

(1) Félix Melchor Santos López ${ }^{(2)}$ Eulogio Santos De la Cruz

\section{RESUMEN}

La logistica inversa es un concepto relativamente nuevo y en los últimos tiempos está adquiriendo más fuerza e interés por parte de las empresas, gobiernos y la sociedad civil, debido al discurso actual de conservación ambiental y el problema del calentamiento global. En la etapa productiva, este concepto está relacionado básicamente a las fallas y mermas producidas en la fabricación de productos, cuyos materiales y materias primas son factibles de ser reutilizados. En este caso, la aplicación de logística inversa interesa especialmente a las medianas y grandes empresas manufactureras, que encuentran la forma conveniente de reducción de costos, la proyección de una imagen de responsabilidad ambiental y la utilización de nuevas tecnologías. Como complemento, se necesitan herramientas automatizadas que permitan ayudar en la toma de decisiones ante fallas del proceso productivo, el cual es el objetivo de esta investigación. Además, se demuestra que su aplicación y resultados conllevan a la ayuda de toma de decisiones correctas a medida que se acumulen datos en el tiempo, es decir, se logre una madurez del sistema.

Palabras clave: Logística inversa, manufactura, fabricación, producción.

APPLICATION OF A MODEL FOR IMPLEMENTING REVERSE LOGISTICS IN THE PRODUCTIVE SLAGE

\section{ABSTRACT}

Reverse logistic is a recently concept that has been taking more importance, force and interest by enterprises, governments and civil society, because currently we are living a peach of environment conservation and reducing of global warming. In the production phase, this concept is linked with mistakes and leftovers made in the fabrication process whose materials are possible of reuse. Thus, the application of reverse logistic is made by medium and large production enterprises, focused in manufacture, that are looking for reduce of costs and give a responsibility environmental image. In addition, enterprises need automated tools for taking good decisions when appear a mistake in the process production, which is the objective of this research. Finally, this system improves the results in the time, when it adds more data.

Key words: Reverse logistic, manufacture, fabrication, production.

\section{INTRODUCCIÓN}

En la actualidad, la población mundial muestra un acelerado crecimiento que conlleva al aumento del consumo de productos manufacturados a gran escala, esto implica un mayor volumen de producción por parte de estas empresas para satisfacer las demandas de la población y de otras empresas.

La logística inversa aparece como una solución factible a los aumentos de producción, ya que su aplicación abarca tres etapas: "reciclaje", "producción" y "devoluciones" (ver figura 1). El reciclaje se da ante la necesidad de recuperar y precisamente reciclar aquellos productos que fueron puestos en venta para su posterior uso por los compradores. En esta etapa, las empresas de producción realizan planes de adquisición de productos desechados para que una vez en planta, se proceda a la separación de los diferentes materiales y, finalmente, pasen a formar parte del stock de materias primas y materiales para la producción. Esta etapa es aplicada por empresas que se dedican a la fabricación de automóviles, aviones, computadoras y electrónicos en general, que se preocupan en los productos que se vendieron años anteriores.

En la etapa de producción, la aplicación de logística inversa se destaca por la maximización del uso de los recursos materiales, esto debido a un adecuado control de fallas en los procesos productivos incluyendo las mermas que siempre están presentes. Cabe mencionar que el uso de mermas y las materias primas de planes fallados es una práctica común en un gran número de industrias, sin embargo no se lleva un registro de las cantidades que se utilizan mediante esta forma y tampoco un costo real.

La tercera etapa, devoluciones, involucra a todo los pasos que componen los retornos de productos terminados y puestos a la venta, es decir de aquellos que fueron vendidos o se encuentran a la venta. Esta etapa de la logística inversa es muy utilizada en las grandes tiendas por departamento que tienen constantes devoluciones por garantías, cambios, devolución del importe monetario, etc. 
Figura 1: Etapas de la logística inversa

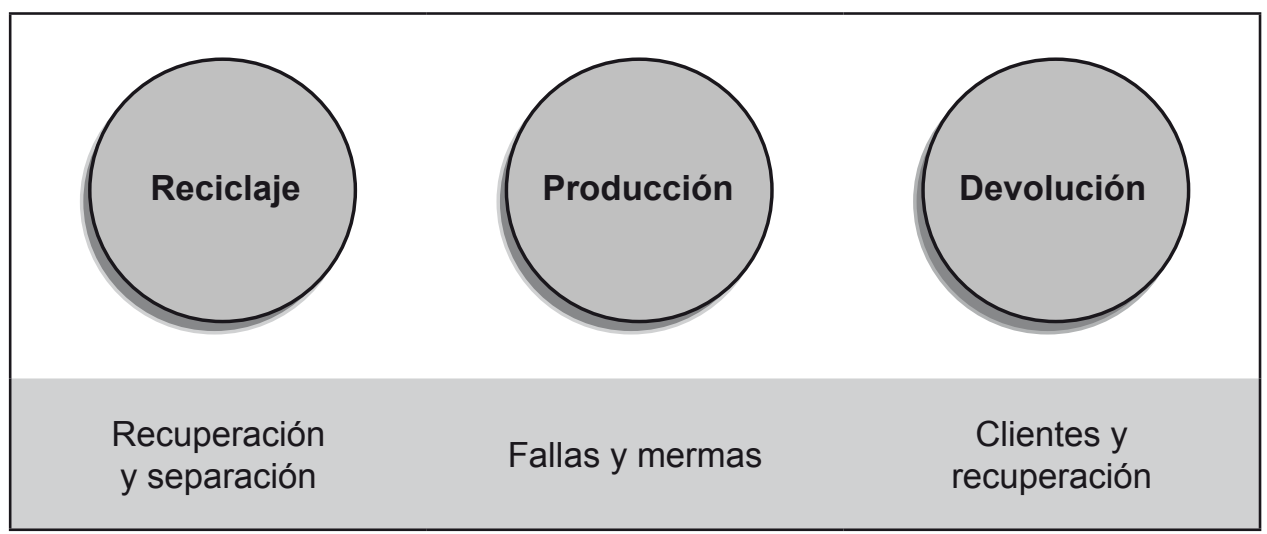

Fuente: Elaboración propia.

\section{DEFINICIÓN}

La actual definición de logística inversa está dada por el Grupo Europeo de Logística Inversa, siendo la más aceptada:

“... El proceso de planeación, implementación y control de flujos de materias primas, inventario en proceso y bienes terminados, desde un punto de uso, manufactura o distribución a un punto de recuperación o disposición adecuada" ${ }^{[1]}$.

Esta definición no tendría sentido sin la relación que existe entre la logística directa o tradicional con la logística inversa (ver figura 2), que comparten mu- chas actividades y procesos comunes así como el uso de herramientas informáticas para sus operaciones. Todo ello enfocado hacia la distribución del suministro.

Para la correcta aplicación de logística inversa, es necesario analizar los principales motivos por los que se dan los retornos de los productos y las principales acciones o decisiones que se suelen tomar ante está situación. Por ello, en el siguiente gráfico, se muestran dos estadísticas, una por las razones de devolución y otra de las decisiones tomadas (gráfico elaborado por el grupo europeo de logística inversa en base a los países miembros de este grupo).

Figura 2: Integración de la logística directa e inversa
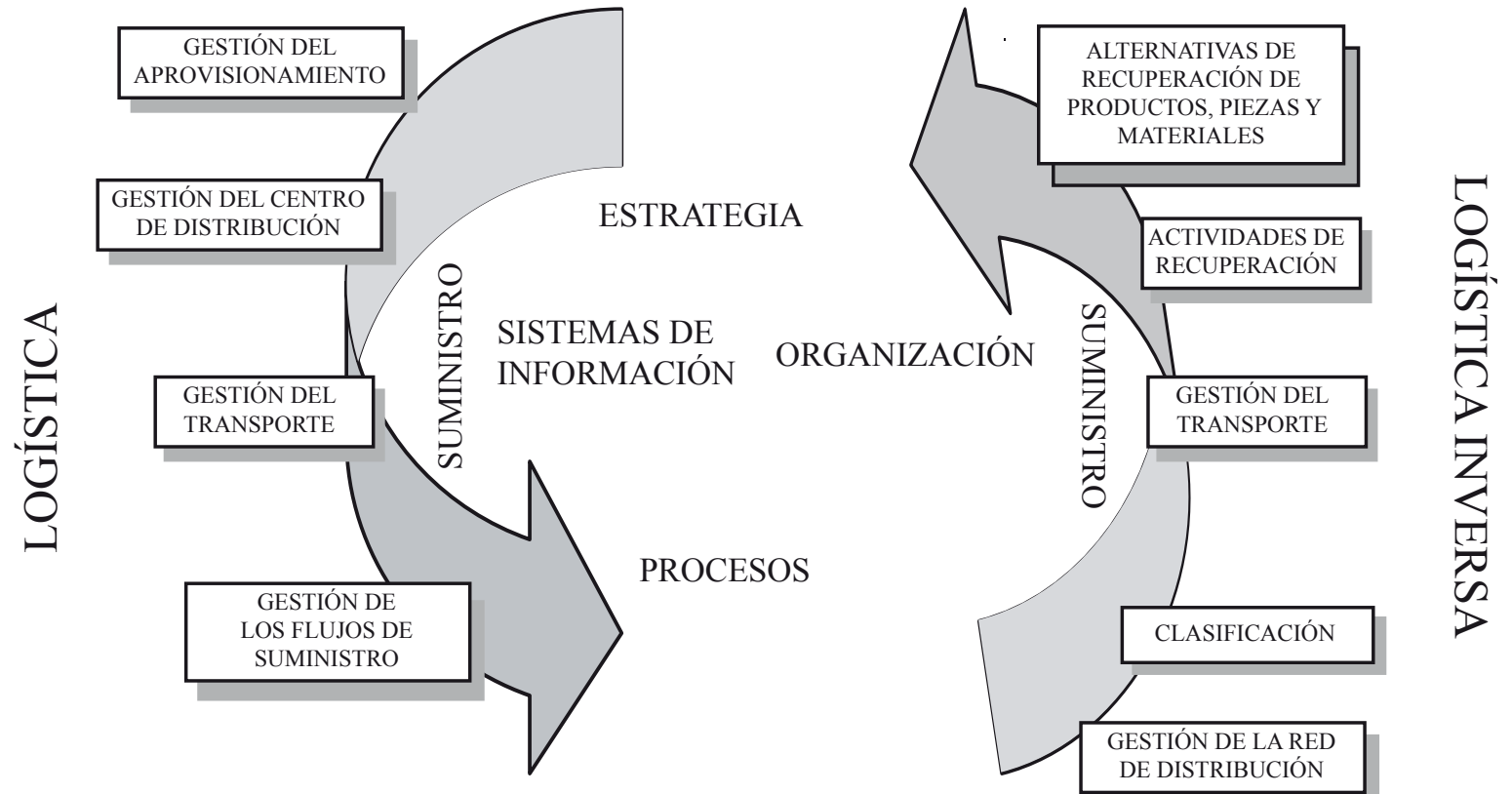

Fuente: tomado de la referencia[ ${ }^{[2]}$ 
Figura 3. Gráfico de devoluciones de productos

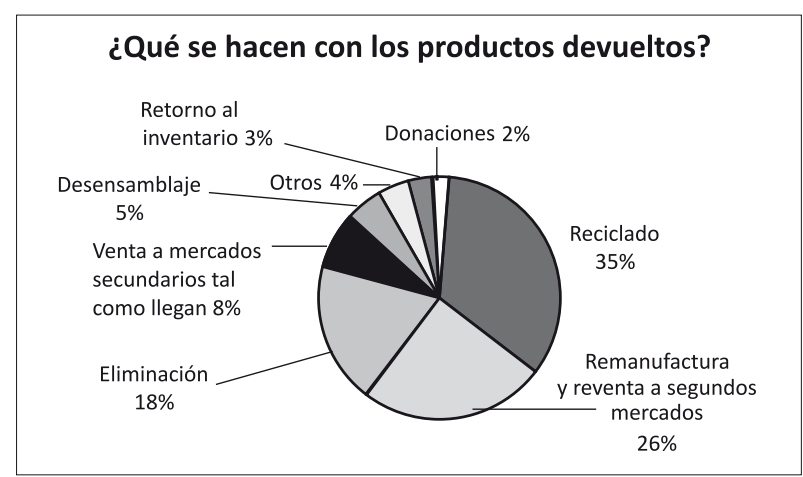

Fuente: Tomado de la referencia ${ }^{[3]}$.

Como se ve en la Figura 3, el mayor porcentaje de devoluciones es por falla de productos en proceso y/o productos terminados (defectuosos); y es ahí a donde apunta el presente trabajo. Es decir, a encontrar una solución automatizada que permita la toma de decisiones en base a que el error o falla que aconteció en el proceso productivo.

\section{CASOS DE ESTUDIO}

A continuación, se mencionarán dos casos reales de la aplicación de logística inversa y un caso de su no aplicación.

\subsection{Caso de una empresa embotelladora de ga- seosa}

Esta empresa, localizada en la ciudad de México, es la cuarta productora más grande de plásticos a nivel mundial. En ella, se implementó un software especializado en logística inversa: Numetrix/3.

"Es una herramienta táctica de planeación, que permite controlar la fabricación de botellas en respuesta a los picos de demandas producidas por promociones, logrando además el ahorro en almacenamiento, debido a que la disponibilidad de botellas pasó a ser conocida por la empresa". [4]

\section{2. caso de una empresa de fabricación de bombas de agua}

En el Perú, existe una empresa líder en la fabricación de bombas centrifugas, helicoidales y piezas fundidas. Dicha organización aplica logística inversa en su proceso de producción y sus compras de materias primas, principalmente.

Las materias primas son compradas en condición de chatarra para después pasar por un proceso de

\section{Razones de devolución de productos}

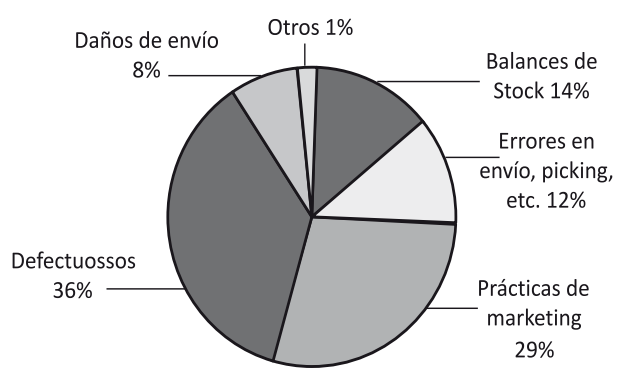

fundición. Es en este proceso de reciclado donde se aplica uno de los puntos de logística inversa, ya que la empresa recurre a materiales existentes y usados, con ello disminuye la adquisición de nuevos insumos.

Por otro lado, en sus procesos de producción, aplica actividades como el de fundición, limado, torneado, taladrado, etc., que generan una gran cantidad de merma acumulada, la cual es aprovechada nuevamente regresando a la etapa de fundición.

Este proceso de logística inversa se repite para cada una de las piezas que forman parte del producto terminado. Por ende, logran un uso máximo de materiales y una importante reducción de costos.

Sin embargo, la empresa registra la cantidad de chatarra que adquirió y no hace una diferenciación de cuánto de esa cantidad fue usada por medio de logística inversa, aunque el sistema de información de la empresa registra muy bien todos los parámetros solo de la logística directa.

\subsection{Caso de la empresa de fabricación de trans- formadores industriales}

Es una empresa peruana dedicada a la fabricación de transformadores industriales, empleando principalmente planchas de flejes magnéticos, acero y hierro.

El peso del transformador más pequeño que fabrica es una tonelada. El proceso de producción incluyen actividades como corte, taladrado, limado, granallado, soldado, etc. La empresa no aplica logística inversa y toda la merma generada y fallas son desechadas, lo cual lleva a un aumento en los costos (compra de más materia prima), así como el aprovechamiento económico de algunos empleados de la empresa con la venta de estas mermas. 
Figura 4. Ejemplo gráfico de un plan de producción dividido en fases (An = Actividad número).

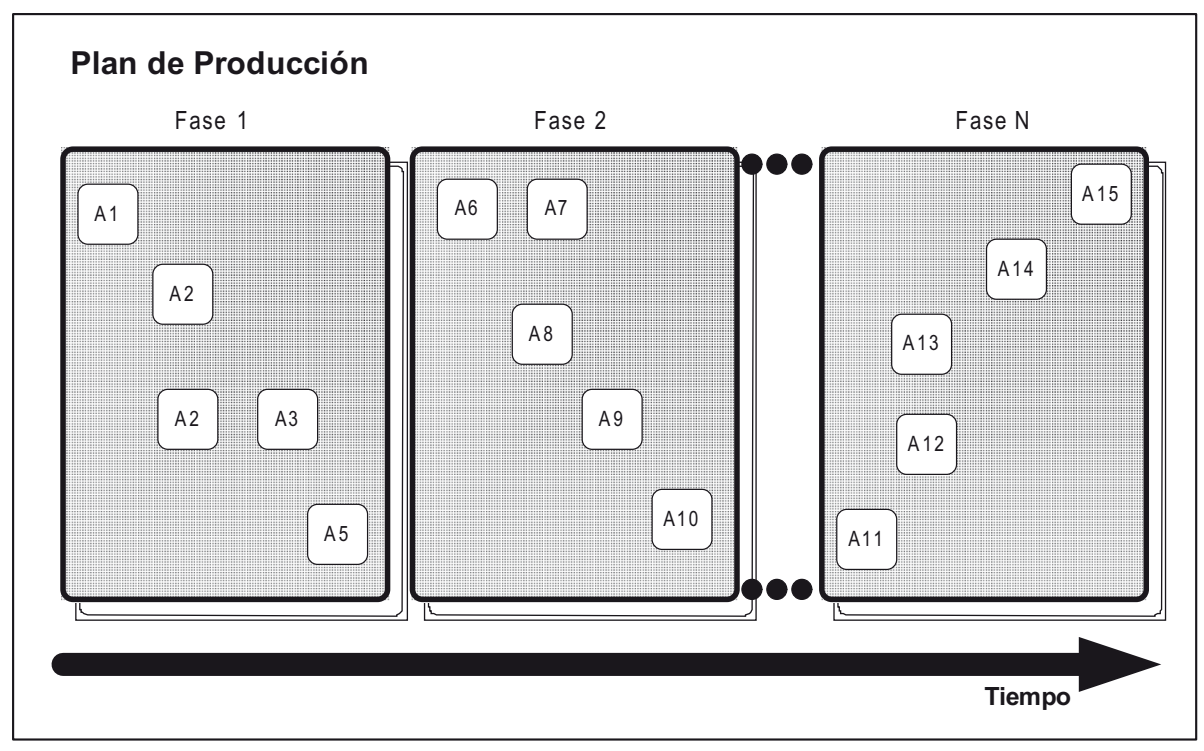

Fuente: Elaboración propia.

La empresa posee un sistema de información de logística directa, mas no de la inversa ya que no lo aplica. Sin embargo, un marco legal más estricto en un futuro, así como ciertas certificaciones ambientales como el caso del ISO 14 000, cambiará la forma de trabajar de esta empresa.

\section{SOLUCIÓN PLANTEADA}

La solución planteada para la implementación de logística inversa en la etapa productiva se detalla de la siguiente forma:

\subsection{Solución del plan de producción}

Se requiere un plan de producción para cada producto, donde se muestren cada una de las actividades, tareas o pasos que lo componen. Se recomienda que estas, a su vez, se agrupen en un conjunto de subprocesos o fases que permiten una mayor abstracción y entendimiento para el caso de planes productivos complejos. Un ejemplo se muestra en la figura

\subsection{Solución de la asignación de costos}

En base a las actividades mostradas de la figura 4, se sugiere la asignación de los costos directos y los costos indirectos.

La asignación de costos directos debe ser a cada actividad, comprendiendo la mano de obra directa y otros costos directos. La asignación de los costos de materia prima se deberá de obtener de un almacén, es decir, se verificará que exista stock suficiente para la fabricación y se irá disminuyendo la cantidad de stock en base al método PEPS (Primero en Entrar Primero en Salir PEPS). Cabe mencionar que se podrá aplicar otro método para la asignación de costos, ya que esta debe ser acorde a la realidad de cada empresa.

Los costos indirectos no son identificables con un producto en particular, es decir, son costos que pueden o no estar en el proceso productivo, sin embargo son necesarios para el funcionamiento y operatividad de la empresa. Por ejemplo, los costos de la fuerza de ventas, la gerencia general, amortizaciones de intangibles, combustibles, etc.

Para la asignación de costos indirectos, es necesario optar por un sistema de costeo específico y acorde con el tipo de producción a realizar. El sistema de costeo indirecto a utilizar será el de "Costeo por órdenes de producción", ello debido a que "las empresas que comúnmente utilizan este sistema son: de impresión, astilleros, aeronáutica, de construcción, de ingeniería” ${ }^{[5]}$.

Una orden de producción estará conformada por líneas de órdenes, donde cada una de ellas reflejará un producto y la cantidad a producir, según el esquema mostrado en la figura 5 . 
Figura 5: Ejemplo de una de orden de producción (ficticia)

\section{Orden N.: 0000001}

$\underline{\text { Nombre del producto }}$

\section{$\underline{\text { Cantidad }}$}

Producto 'A'

Producto 'B'

Producto ' $\mathrm{C}$ '

-....

….

Producto ' $\mathrm{X}$ '

Fuente: Elaboración propia

Para la distribución o prorrateo de los costos indirectos, será necesario utilizar una base de asignación o inductor, los cuales podrán ser los siguientes:

- Unidades de producción

- Horas máquina

- Costo de mano de obra directa

Los inductores se calcularán con la división de lo que le corresponde a cada producto entre el total.

$$
\begin{aligned}
& \operatorname{ind}_{\varphi_{x}} \equiv \frac{\text { Valor_del_Producto }}{\text { Producto }_{-} X^{\prime}} \\
& \sum_{i=\text { Producto }_{-} A^{\prime}} \text { Valor_del_Producto }_{i}
\end{aligned}
$$

Siendo:

$$
\sum_{i=\text { Producto' } A^{\prime}}^{\text {Producto }} \mathrm{A}^{\prime}
$$

La sumatoria del valor de todos los productos de la orden de producción.

$$
\operatorname{ind} \varphi_{x}
$$

El valor del inductor para un producto determinado, obtenido mediante la división del valor del producto entre la sumatoria de todos los productos.

Además, después de calcular el inductor correspondiente a cada producto se procederá a distribuir el costo indirecto para cada producto.
Siendo:

CostolndirectoTotal

El costo indirecto total para la orden de producción.

Costo_Indirecto_Producto ${ }_{x}$

El costo indirecto para un producto determinado

Para costear los costos indirectos solo se utilizará un inductor por cada orden de producción.

\subsection{Aplicación de logística inversa en produc- ción}

La aplicación de logística inversa en la etapa de producción consiste en la recuperación de materia prima que se generan por fallas (ver figura 4). En tal caso y en base a las ordenes de producción, se deberán de almacenar las distintas fallas producidas para los productos, así como las decisiones que se tomaron con respecto a estas, por ejemplo: reciclar, re manufacturar, incinerar, etc.

\subsection{Modelo para cálculo de la mejor decisión}

"El proceso de jerarquía analítica (AHP, por sus siglas en inglés) está diseñado para casos en que las ideas, sentimientos y emociones se cuantifican con base en juicios subjetivos para obtener una escala numérica para dar prioridades a las alternativas de decisión" [6].

En base a lo citado en los puntos anteriores, se pretende mostrar una solución para el cálculo de la mejor decisión a tomar en base a la(s) falla(s) producida(s) en las órdenes de producción.

El proceso de jerarquía analítica está compuesto de tres partes y se aplicarán de la siguiente manera:

- Decisión. Es el objetivo del proceso, que en este caso, es el cálculo de la mejor decisión a tomar.

- Criterio de Jerarquía. Es aquel juicio o criterios para el cálculo de la decisión. Para este punto, se escogieron cuatro criterios de jerarquía, los cuales son:

- Frecuencia de la decisión tomada en el producto para una actividad en particular.

- Frecuencia histórica en que la decisión fue tomada.

- Costo de recuperación logrado con la toma de la decisión para el producto y la actividad específica. 
Ejemplo de la matriz para la aplicación del proceso de jerarquía analítica.

\begin{tabular}{|c|c|c|c|c|}
\hline Decisión & $\begin{array}{l}\text { Frecuencia en el } \\
\text { producto }\end{array}$ & $\begin{array}{l}\text { Frecuencia } \\
\text { histórica }\end{array}$ & $\begin{array}{c}\text { Costo de } \\
\text { recuperación } \\
\text { en el producto }\end{array}$ & $\begin{array}{l}\text { Costo de } \\
\text { recuperación } \\
\text { histórica }\end{array}$ \\
\hline $\begin{array}{l}\text { Decisión } 1 \\
\text { (Re venta) }\end{array}$ & $x x$ & $\mathrm{~mm}$ & kk & $\mathrm{mm}$ \\
\hline $\begin{array}{l}\text { Decisión } 2 \\
\text { (Fundir) }\end{array}$ & yy & $x x$ & ZZ & zZ \\
\hline $\begin{array}{l}\text { Decisión } 3 \\
\text { (Re manufacturar) }\end{array}$ & ZZ & yy & $x x$ & yy \\
\hline $\begin{array}{l}\text { Decisión } 4 \\
\text { (Desemsamblar) }\end{array}$ & $\mathrm{mm}$ & $\mathrm{mm}$ & $\mathrm{pp}$ & $x x$ \\
\hline $\begin{array}{l}\text { Decisión } 5 \\
\text { (Desechar) }\end{array}$ & kk. & $\mathrm{pp}$ & kk & $\mathrm{mm}$ \\
\hline Decisión N & \multirow{2}{*}{ th } & \multirow{2}{*}{ kk } & \multirow{2}{*}{ yy } & \multirow{2}{*}{$X X$} \\
\hline (Desechar) & & & & \\
\hline
\end{tabular}

Fuente: Elaboración propia.

- Costo de recuperación histórica lograda con la toma de la decisión.

- Alternativas. Son todas aquellas posibles decisiones a tomar, es decir, es la lista de las cuales se escogerá a la mejor según el cálculo.

Para realizar el cálculo se establecerá una matriz de dos dimensiones como se muestra en la tabla 1.

La matriz permite asignar un peso porcentual a cada "criterio de jerarquía", siendo esta de $25 \%$ para cada una ellas por defecto. Este porcentaje es modificable y quedará a criterio de la persona que lo aplique, en base a su juicio y experiencia.

Asimismo, los valores de cada "jerarquía de decisión" y para cada decisión, deberán ser extraídas de la data acumulada en una base de datos, es decir, se basará en datos históricos que se van acumulando en el tiempo, los cuales permitirán un cálculo de mejor calidad. Estos datos podrán ser valores enteros o decimales, ya que son obtenidos precisamente de un cálculo.

Finalmente, la fórmula para el cálculo de la mejor decisión será como sigue: $\beta_{\text {Decisiónl }} \equiv 0.25 \times x x+0.25 \times k k+0.25 \times \mathrm{mm}$

$\beta_{\text {Decisión } 2} \equiv 0.25 x y y+0.25 x x x+0.25 x z z+0.25 x z z$

$\beta_{\text {Decisión } 3} \equiv 0.25 \times z z+0.25 \times x x+0.25 \times x x+0.25 \times m m$

Se calculará un valor $\beta$ para cada una de las decisiones, siendo la de mayor valor la mejor decisión a tomar, es decir, la respuesta.

El peso de 0.25 se seleccionó debido a que se considera por igual el aporte de cada criterio de jerarquía. Este peso será modificable acorde a la realidad de cada empresa.

\section{SIMULACIÓN DEL PROCESO}

A continuación, se muestra los resultados "gráficos de datos cuantitativos" ${ }^{[7]}$ de la simulación de la aplicación de logística inversa, mediante la utilización de un sistema de información desarrollado a me$\operatorname{dida}^{[2]}$ y que permite el registro y el cálculo de la mejor decisión, en base a los datos históricos registrados. 
Tabla 2. Tabla de valores obtenidos en cada corrida (se muestran solo las 8 primeras).

\begin{tabular}{|l|l|l|l|l|l|l|l|l|}
\hline \multicolumn{1}{|c|}{$\begin{array}{c}\text { N. } \\
\text { Decisión }\end{array}$} & \multicolumn{1}{c|}{} & \multicolumn{1}{c|}{$\mathbf{2}$} & $\mathbf{3}$ & \multicolumn{1}{c|}{$\mathbf{4}$} & \multicolumn{1}{c|}{$\mathbf{5}$} & $\mathbf{6}$ & $\mathbf{7}$ & $\mathbf{8}$ \\
\hline Reventar & 0.23 & 0.17 & 0.16 & 0.05 & 0.04 & 0.02 & 0.01 & 0.01 \\
\hline Fundir & 0.25 & 0.28 & 0.36 & 0.4 & 0.52 & 0.71 & 0.89 & 0.91 \\
\hline Remanufacturar & 0.36 & 0.25 & 0.15 & 0.1 & 0.1 & 0.14 & 0.08 & 0.06 \\
\hline Desensamblar & 0.11 & 0.12 & 0.1 & 0.35 & 0.24 & 0.12 & 0.01 & 0.01 \\
\hline Desechar & 0.05 & 0.18 & 0.23 & 0.1 & 0.1 & 0.01 & 0.01 & 0.01 \\
\hline Total & 1 & 1 & 1 & 1 & 1 & 1 & 1 & 1 \\
\hline
\end{tabular}

Fuente: Elaboración propia

Figura 6: Gráfica

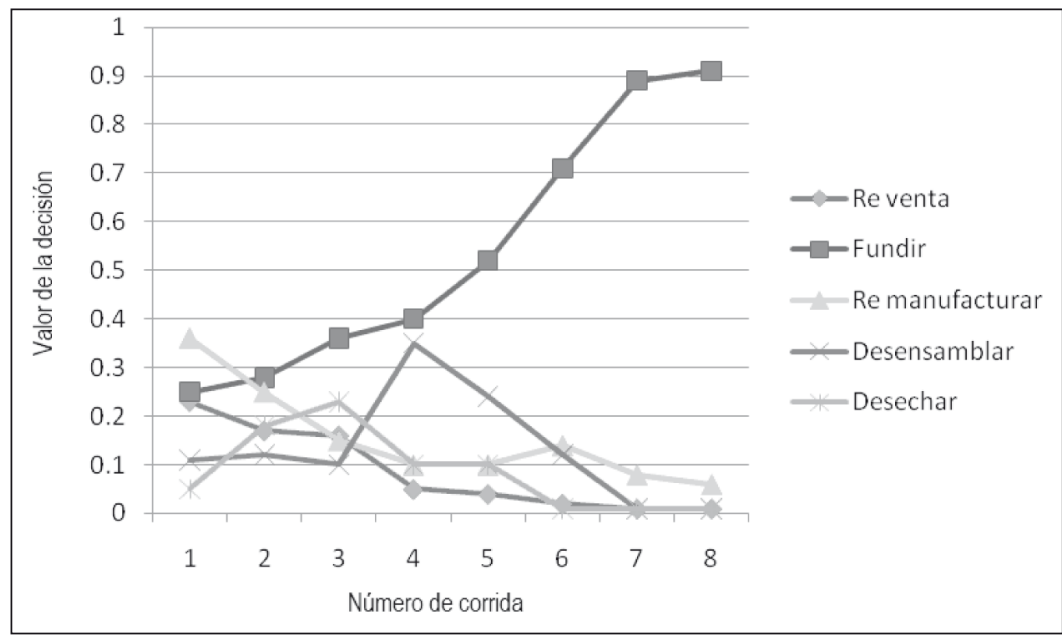

Fuente: Elaboración propia

Producto: Bomba Centrifuga 3500 rpm, 75 HP. Actividad: Taladrar.

Decisiones: Se muestran los más representativos y cuya probabilidad es superior o igual a 0.1 (redondeado a 2 decimales).

Número de corridas entre cada intervalo: 30 (cantidad estándar y adecuada en estadística) ${ }^{[7]}$.

En la Tabla 2, se muestran los valores Beta calculados para cada actividad en cada una de las corridas. Cada una de las corridas, se realiza en diferentes tiempos. Es decir, que a medida que se toma cada corrida se aumentaron la cantidad de datos en el sistema de información, con ello se va ajustando cada vez más los valores.
Como se observa en la Figura 6, con el transcurrir del tiempo y uso del sistema, se aumentan los datos para el cálculo del proceso de jerarquía analítica; por ende, se podrá obtener una tendencia de la mejor decisión a tomar ya que se acerca a un valor cercano a $1(100 \%)$.

\section{CONCLUSIONES}

Se espera que la aplicación de logística inversa en la etapa productiva sea realizado por empresas industriales, por ello conviene alimentar los datos para el cálculo de la toma de decisiones en base a un Sistema de Gestión de Calidad ISO 9001; por ejemplo, ya que esta norma exige el registro de los puntos fuera de control (fallas) y el registro de las acciones correctivas (decisiones). 
Si no se cuentan con datos registrados suficientes (data histórica) en un primer momento, se debe de esperar a encontrar la madurez y registro de estos datos, de este modo se garantiza un correcto y mejor cálculo del Proceso de Jerarquía Analítica.

Finalmente, de la simulación del proceso desarrollado en el punto 5, se deduce que, a medida que transcurre el tiempo, es decir, se aplique logística inversa y registre sus datos históricos, se podrá calcular la mejor decisión a cierta falla producida en una determinada actividad. Todo ello, en virtud de que la tendencia de esta mejor decisión va tomando un valor cercado a 1 , que se aprecia claramente con el incremento del número de corridas.

\section{REFERENCIAS BIBLIOGRÁFICAS}

1. RevLog. Definición actual del concepto de logística inversa por parte del "grupo europeo de logística inversa". Disponible en:

http://www.reverselogisticstrends. com/reverselogistics.php (visitado el 10-06-2010).

2. Carrefur. (2001). "La implantación de la logística inversa en una multinacional de la distribución.
Experiencia Carrefour". Foro Internacional Pilot. Zaragoza España. 7-8.

3. Rubio Lacoba, S. y T. Bañegil. El diseño de la función inversa de la Logística: Aspectos estratégicos, tácticos y operativos. XI Congreso ACEDE.

4. Cadwell Bruce. (1999). "Untapped opportunities exist in returned products, a side of logistics few businesses have thought about - until now", Information Week 729.

5. Rasgado C. Artículo de conceptos y definiciones de órdenes de producción en empresas de producción. Instituto Tecnológico del Istmo, México. Disponible en:

http://www.itistmo.edu.mx/pagina $\% 20$ de $\% 2$ Ola $\% 20$ lic\%20.Cecilia\%20Rasgado\%20Luis/ COSTOS\%20I\%20UNI5.html (visitado el 13-052008).

6. Taha H. A. (2004). Investigación de Operaciones. 7. ${ }^{a}$ ed. Pearson, ISBN: 9702604982, México.

7. Mendenhall W. (1997). Probabilidad y Estadística para Ingeniería y Ciencias. Prentice Hall, ISBN: 968-880-960-8, México. 